\title{
Prostaglandin insert dinoprostone versus trans-cervical balloon catheter for outpatient labour induction: a randomised controlled trial of feasibility (PROBIT-F)
}

Amarnath Bhide ${ }^{1,2,3^{*}}$ D. Philip Sedgwick ${ }^{2}$, Barbara Barrett ${ }^{4}$, Georgina Cupples ${ }^{1}$, Rose Coates ${ }^{5}$, Rosie Goode ${ }^{6}$, Sandra Linton ${ }^{1}$ and Christine McCourt ${ }^{5}$

\begin{abstract}
Background: The aim was to assess the feasibility of conducting a randomised controlled trial $(R C T)$ of induction of labour comparing use of two methods in the outpatient setting.

Methods: An open-label feasibility RCT was conducted in two UK maternity units from October 2017 to March 2019. Women aged $\geq 16$ years, undergoing induction of labour (IOL) at term, with intact membranes and deemed suitable for outpatient IOL according to local guidelines were considered eligible. They were randomised to cervical ripening balloon catheter (CRB) or vaginal dinoprostone (Propess). The participants completed a questionnaire and a sub-group underwent detailed interview. Service use and cost data were collected via the Adult Service Use Schedule (AD-SUS). Women who declined to participate were requested to complete a decliners' questionnaire.

Results: During the study period, 274 eligible women were identified. Two hundred thirty (83.9\%) were approached for participation of whom 84/230 (36.5\%) agreed and 146 did not. Of these, 38 were randomised to Propess $(n=20)$ and CRB $(n=18)$. Decliner data were collected for 93 women. The reasons for declining were declining IOL $(n=22)$, preference for inpatient IOL $(n=22)$ and preference for a specific method, Propess $(n=19)$. The intended sample size of 120 was not reached due to restrictive criteria for suitability for outpatient IOL, participant preference for Propess and shortage of research staff.

The intervention as randomised was received by 29/38 (76\%) women. Spontaneous vaginal delivery was observed in $9 / 20$ (45\%) women in the dinoprostone group and 11/18 (61\%) women in the CRB group. Severe maternal adverse events were recorded in one woman in each group. All babies were born with good condition and all except one $(37 / 38,97.4 \%)$ remained with the mother after delivery. No deaths were recorded. $-21 \%$ of women in the dinoprostone group were re-admitted prior to diagnosis of active labour compared to $12 \%$ in the CRB group.

(Continued on next page)
\end{abstract}

\footnotetext{
* Correspondence: abhide@sgul.ac.uk

${ }^{1}$ Fetal Medicine Unit, St. George's University Hospital Foundation Trust,

Blackshaw Road, London SW17 OQT, UK

${ }^{2}$ St George's, University of London, London, UK

Full list of author information is available at the end of the article
}

C C The Author(s). 2020 Open Access This article is licensed under a Creative Commons Attribution 4.0 International License, which permits use, sharing, adaptation, distribution and reproduction in any medium or format, as long as you give appropriate credit to the original author(s) and the source, provide a link to the Creative Commons licence, and indicate if changes were made. The images or other third party material in this article are included in the article's Creative Commons licence, unless indicated otherwise in a credit line to the material. If material is not included in the article's Creative Commons licence and your intended use is not permitted by statutory regulation or exceeds the permitted use, you will need to obtain permission directly from the copyright holder. To view a copy of this licence, visit http://creativecommons.org/licenses/by/4.0/ The Creative Commons Public Domain Dedication waiver (http://creativecommons.org/publicdomain/zero/1.0/) applies to the data made available in this article, unless otherwise stated in a credit line to the data. 
(Continued from previous page)

Conclusions: A third of the approached eligible women agreed for randomisation. An RCT is not feasible in the current service context. Modifications to the eligibility criteria for outpatient $\mathrm{OL}$, better information provision and round the clock availability of research staff would be needed to reach sufficient numbers.

Trial registration: NCT03199820. Registered on June 27, 2017

Keywords: Labour, induced, Cervical ripening, Randomised controlled trial, Cook cervical ripening balloon, Outpatients, feasibility

\section{Key messages}

- The study is not feasible using existing eligibility criteria for outpatient induction of labour (IOL); further modifications to the eligibility criteria for outpatient IOL would be needed to make it feasible.

- Other reasons for the low recruitment rate were participant preference for prostaglandin pessary and shortage of research staff.

- No major adverse effects were recorded attributible to the outpatient setting for induction of labour in this small sample.

\section{Introduction}

Over the last decade, the rate of induction of labour (IOL) in England has increased steadily to around 30\% of all pregnant women [1]. Currently, most women undergoing induction of labour are admitted to the hospital prior to commencing IOL. A Cochrane review assessing methods of outpatient labour induction (cervical ripening or priming) concluded that it was feasible for labour to start at home. However, there is limited evidence as to which induction methods are preferred by women or the interventions that are most effective and safe to use in outpatient settings [2]. A Cochrane review reported that mechanical methods (trans-cervical balloon catheter) of cervical ripening for IOL are as effective as vaginal prostaglandin $\mathrm{PGE}_{2}$ [3]. The UK Database of Uncertainties about the Effects of Treatments (UK DUETs) identified mechanical methods of labour induction as a known uncertainty and recommended that future studies on mechanical methods for IOL should have larger sample sizes and report on substantive outcomes. In a randomised controlled trial [4], 101 women with an unfavourable cervix requiring IOL at term were randomised to outpatient care using Foley catheter or inpatient care using vaginal $\mathrm{PGE}_{2}$. The authors reported that the outpatient group had shorter hospital stay prior to birth whilst vaginal birth rates, total induction to delivery time and total inpatient times were similar. Another trial showed that, for women with an unfavourable cervix at term, success of induction of labour with a mechanical method is similar to induction of labour with progstaglandins, with fewer maternal and neonatal side effects, but similar caesarean section rates [5]. Furthermore, Pennell et al. [6] reported lower pain scores with the use of mechanical method as compared to prostaglandins. Both studies were apparently undertaken in an inpatient setting. The OPRA study [7] compared clinical outcomes from outpatient with inpatient prostaglandin treatment for low risk labour induction. They concluded that uterine stimulation following prostaglandins may preclude a woman from going home or remaining at home overnight and may not be the best agent for outpatient ripening. Therefore, it would be beneficial to compare outpatient outcomes of prostaglandin treatment with mechanical methods including economic analysis to determine the most suitable agent. The prostaglandin method is the standard practice for IOL at St. George's Hospital, London, and Medway Hospital, Kent. Although mechanical methods are used in some UK hospitals, outpatient use is not common. The Hospital Episode Statistics (HES) database does not record the exact method of induction of labour nor collect data on efficacy, cost-effectiveness, hospital stay or outcome of labour induction stratified according to the method of induction of labour. Therefore, there is no readily available data source that can be used to obtain information on the outcomes of induction of labour using mechanical methods in the outpatient setting. A recent Cochrane review [3] concluded that future research could be focused more on safety aspects for the neonate and maternal satisfaction.

A feasibility trial was deemed necessary before embarking on a randomised controlled trial. It would permit identification of suitable clinical outcome measures with sufficient precision and help design a future randomised controlled trial. The primary objective, therefore, was to investigate the feasibility of conducting a randomised controlled trial of induction of labour using trans-cervical ballon catheter versus vaginal prostaglandin $\mathrm{E}_{2}$ pessary in the outpatient setting.

\section{Methods}

\section{Study design}

We conducted an open-label feasibility RCT (Registration Number: NCT03199820) with sustained-release prostaglandin vaginal pessary (Propess) or cervical ripening balloon catheter (CRB) in the outpatient setting using a 1:1 allocation ratio. The trial took place in two 
UK maternity units: St George's University Hospitals NHS Foundation Trust, South London (October 2017 to March 2019) and Medway University Hospital, Kent (February 2018 to October 2018). The two sites differed in social demographics and were included to enhance the external validity of the results. The study description is publicly available at the clinical trials registry [8]. The trial was approved by the East of England - Cambridgeshire and Hertfordshire Research Ethics Committee (17/ $\mathrm{EE} / 0295)$. The primary objective was to assess the feasibility of conducting a randomised controlled trial (RCT), namely, the number of women willing to enrol. Secondary objectives were to identify suitable clinical outcome measures, estimate service costs and monitor safety, as well as to determine women's willingness to be randomised, to determine the acceptability of using the balloon catheter, to examine women's views on outpatient induction of labour and to assess women's experience with these methods and their preference. Assessment of women's experience with these methods and their preference was through interviews and qualitative analysis and will be reported elsewhere.

\section{Participants}

Inclusion criteria were women aged $\geq 16$ years, undergoing IOL at $\geq 37$ weeks' gestation, with intact membranes, able to give informed consent and deemed suitable for outpatient IOL according to local guidelines. Written information was provided to women regarding the available methods: IOL with sustained release dinoprostone (Propess), or cervical ripening balloon (CRB), both in the outpatient setting. Research teams at each site approached women to confirm eligibility and provided verbal and written information. Strict eligibility criteria have been developed for suitability of outpatient IOL against which research midwives screened for eligible participants and a medical practitioner confirmed that eligibility was met. At both the recruiting sites, the pregnancy had to be uncomplicated at or beyond $41^{+0}$ weeks with a single foetus in cephalic presentation with no risk factors. Trained clinician obtained written informed consent. During the study, the investigators noticed a shortfall of eligible women. Therefore, inclusion criteria were widened in 2018 at St. George's Hospital to include women requiring induction of labour at term $\left(37^{+0}\right.$ weeks or more) with diet-controlled gestational diabetes, who were originally excluded.

\section{Randomisation and masking}

Eligible participants were randomly allocated with a 1:1 ratio to receive Propess or CRB. Randomisation was stratified by site and parity using variable block sizes (two and four). A research team member entered baseline data on a web-based database at study enrolment and then allocated the treatment (Propess or CRB) using the web-based randomisation programme developed by the King's Clinical trial Unit (KCTU). Nature of the intervention mandated that trial participants, clinical care providers or outcome assessors could not be blinded to trial allocation. The statistician was not aware of the allocation sequence and discussions as regards recruitment and was not involved in any of the women's care or recording of their results. The data were supplied to the statistician by the Clinical Trials Unit with the group allocation coded. The group allocation was only revealed following compilation of the results in tabular form.

\section{Procedures}

After randomisation, a member of the research team administered the treatment method according to the recommendations of the manufacturer, described briefly as follows: for induction of labour with Propess, $10 \mathrm{mg}$ insert was introduced in the posterior vaginal fornix close to the cervix, using only small amounts of water-soluble lubricants to aid insertion. The woman was advised to be recumbent for 20-30 min following insertion. For IOL with balloon catheter, the woman was positioned in the dorsal position and a vaginal speculum was inserted to gain cervical access. The cervix was cleaned appropriately to prepare for device insertion. The CRB was inserted into the cervix and advanced until both balloons entered the cervical canal. The uterine balloon was inflated with $40 \mathrm{ml}$ sodium chloride $(0.9 \%)$. Once the uterine balloon was inflated, the device was pulled back until the balloon was against the internal cervical os. The vaginal balloon was now inflated with $20 \mathrm{ml} \mathrm{NaCl}(0.9 \%)$. The speculum was removed after the balloons were situated on each side of the cervix and the device was securely in place. More fluid was added to each balloon in turn, in $20 \mathrm{ml}$ increments until each balloon contained $80 \mathrm{ml}$ (maximum volume of fluid). Following this, clinical care was provided by clinical healthcare practitioners.

Women underwent monitoring of foetal condition and uterine activity by cardiotocography (CTG) according to the local protocol. CTG was discontinued once it was judged to be normal and the woman could go home. She was instructed to return to the hospital at an agreed time on the following morning, if the balloon catheter was spontaneously expelled or if she thought she was in labour, whichever was earlier. On the following morning/upon onset of labour, the device (Propess or CRB) was removed, and artificial rupture of membranes (ARM) attempted (unless spontaneous rupture had occurred already).

The intended recruitment target was randomisation of 120 women between the two sites over a 12-month period. Ability to recruit the intended sample size was considered the demonstration of feasibility. 


\section{Sample size}

The study was designed as a feasibility trial, and as suggested by NIHR guidelines [9], no formal sample size calculation was performed. It has been recommended that the total sample size for a feasibility trial can be between 70 (with allocation to treatments groups in a 1:1 ratio, i.e. 35 per group) if the outcome for the definitive RCT is normally distributed and a total of at least 120 subjects (60 per group) for binary outcomes [10]. It was important to allow for incomplete data and protocol violation. Therefore, it was thought more efficient to use the larger sample size to guard against the lack of precision by using inflated estimates. Therefore, the intended sample size would provide information on the primary outcome measures with sufficient accuracy to inform a power calculation for the definitive randomised controlled trial.

Women who declined to participate in the trial were invited to complete a short questionnaire exploring their main reasons for not participating. Verbal feedback was obtained for those who declined to complete the questionnaire. After they had given birth, the participants were asked to complete a questionnaire within $48 \mathrm{~h}$. The questionnaire was modified from the one used in a previously published study [4]. This recorded women's experience and acceptability of the two methods. All women who took part in the RCT were also invited to participate in a semi-structured interview at least 4 weeks after the birth. Partners were also invited with the women's permission. The detailed methods and findings of the interviews as well as the post-natal questionnaire will be reported in a separate paper.

Participant demographics and clinical and patientreported data were collected using an online database developed by KCTU. The clinical and patient-reported data included vital signs at trial entry, cervical Bishop score, birth details, maternal and foetal outcomes including adverse outcomes, use of pain relef measures at home and in the hospital, survey responses and decliner questionnaire responses.

The Adult Service Use Schedule (AD-SUS) was used to collect service use data [11]. It is a researchercompleted questionnaire, adapted for use in this study following a review of relevant and in collaboration with the clinical research team. The AD-SUS was completed using data from electroinc hospital records. Since this was a feasibility study, the usefulness of the instrument was judged by the ease of completion by the research staff and its ability to provide the data needed to generate costs for a full economic evaluation. The method for estimating the cost of the alternative interventions for this study required work in a feasibility stage because of the need to capture all aspects of induction. Service use data were reviewed in order to establish the most accurate approach to estimating the cost and alternative methods were compared. The options for sources of unit costs for the intervention and associated resources were also explored, making use of nationally available costs and optimising links with the service use data.

Research teams undertook standard assessments of safety, with reporting of adverse events and serious adverse events following usual governance procedures for a clinical trial of an investigational medicinal product overseen by the UK Medicines and Healthcare Regulatory Agency (MHRA).

\section{Statistical analysis}

The analysis and presentation of results follow the CONSORT guidelines [12] (Fig. 1). All analyses followed the intention-to-treat principle: all randomly allocated women (and infants) were analysed according to the group they were allocated to, irrespective of the intervention they received. Demographic and clinical data were presented as frequencies and percentages for categorical variables and mean and standard deviation for normally distributed continuous variables. Statistical hypothesis testing was not performed since this was a feasibility study.

\section{Role of funding source}

The funder of the study had no role in study design, data collection, data analysis, data interpretation or writing of the report. The corresponding author had full access to all the data in the study and had final responsibility for the decision to submit for publication.

\section{Results}

\section{Feasibility outcomes}

Between October 16, 2017, and March 31, 2019 (18 months), 3293 women (2167 at St George's and 1126 at Medway) underwent IOL. Out of these, 274 women (8.32\%) were found to be eligible for inclusion according to local criteria for outpatient IOL. Of the 274 eligible women, 230 (83.9\%) were approached for participation of whom 84 (36.5\%) agreed (two/month/site). Of these, 46 women were excluded for reasons including ARM and delivery by IOL date. The remaining 38 women were randomised: Propess $(n=20)$, CRB $(n=18)$. Of the 146 women not agreeing to participate, 93 completed a questionnaire. Reasons for declining participation (please see Table 1) included declining IOL $(n=$ $22)$, preference for inpatient IOL $(n=22)$ or for a specific method (Propess, $n=19$ ). Of those randomised women, nine (24\%) did not receive the intervention they were originally allocated (details in the Consort Flowchart). Feasibility outcomes according to the participating centre are shown in Table 2. 


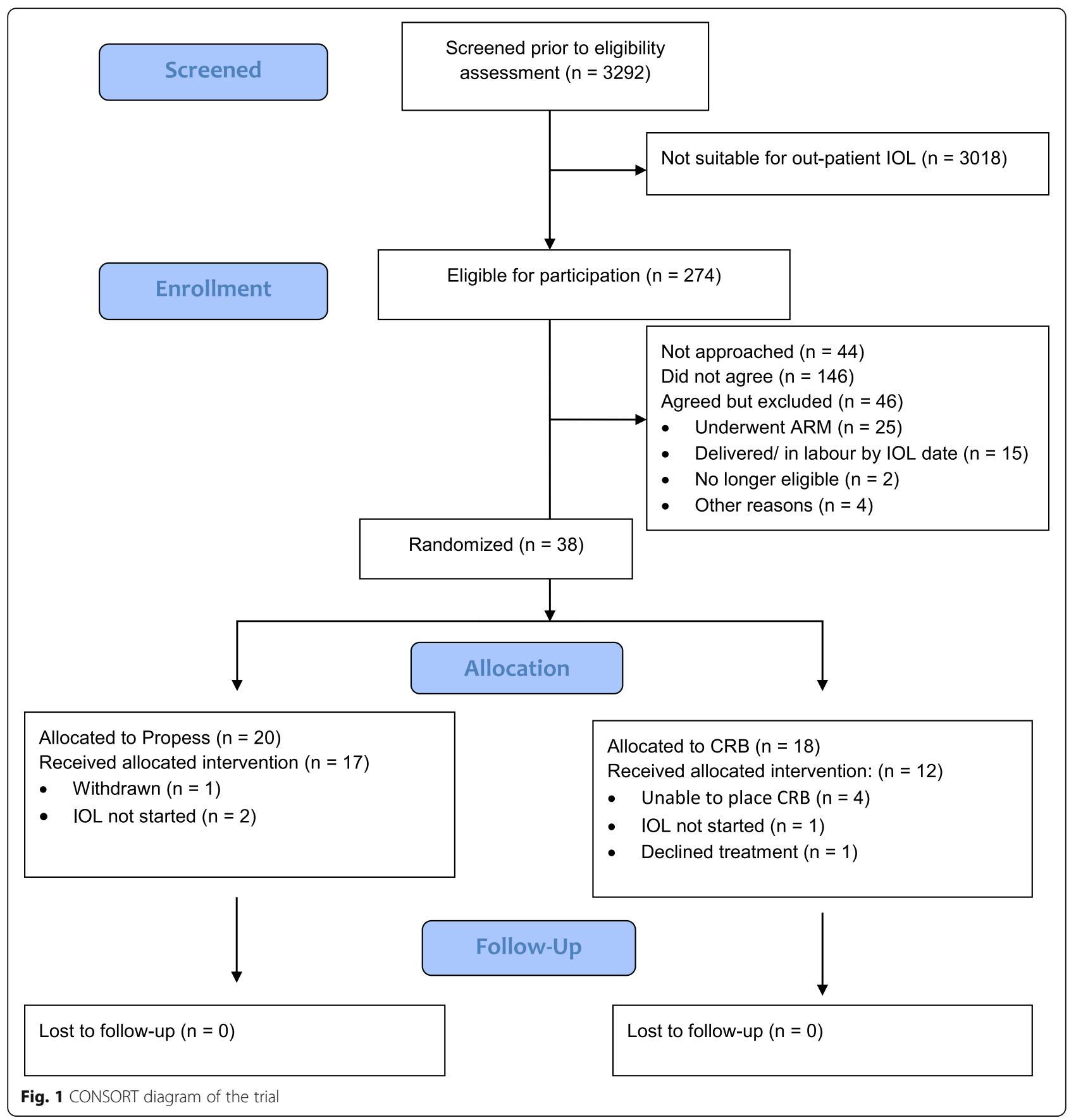

Table 1 Decliners' information

\begin{tabular}{ll}
\hline & No. of women \\
\hline Total women declining participation & 117 \\
Decliners' data available & 93 \\
Verbal response & 70 \\
Decliners' questionnaire & 23 \\
Main reason for declining & \\
Preferring inpatient IOL & $22 / 93$ \\
Declining IOL by any method & $22 / 93$ \\
Preferring Propess & $19 / 93$ \\
\hline
\end{tabular}

\section{Clinical characteristics}

Participant demographics at randomisation are shown in Table 3. The women had a mean height of $168.9 \mathrm{~cm}$, mean weight $69.1 \mathrm{~kg}$ and mean BMI $24.2 \mathrm{~kg} / \mathrm{m}^{2}$, whilst 25/38 (65.8\%) were nulliparous. The majority of women 29/38 (76\%) were of white European ethnicity. The mean age of women in the Propess group was 34.1 years, compared to 33.2 years in the CRB group.

Maternal and foetal parameters at baseline are shown in Table 4. Maternal vital signs were within the 
Table 2 Feasibility outcomes by centre and for all participants

\begin{tabular}{llll}
\hline & SGH & Medway & All participants \\
\hline Women delivering in the study period $(n)$ & 2167 & 1126 & 3292 \\
Number of eligible participants $(n, \%)$ & $168(7.75 \%)$ & $106(9.41 \%)$ & $274(8.32 \%)$ \\
Number of participants approached $(n, \%)$ & $156(92.9 \%)$ & $74(69.8 \%)$ & $230(83.9 \%)$ \\
Participants randomised $(n, \%)$ & $30(19 \%)$ & $6 / 8(75.0 \%)$ & $38(16.5 \%)$ \\
Received allocated intervention $(n, \%)$ & $23 / 30(76.7 \%)$ & $2 / 8(25.0 \%)$ & $29 / 38(76.3 \%)$ \\
Did not receive allocated intervention $(n, \%)$ & $7 / 30(23.3 \%)$ & 1 & $8 / 38(21.1 \%)$ \\
Withdrew from trial intervention $(n)$ & 0 & $5(62.5 \%)$ & 1 \\
Complete data available $(n, \%)$ & $23(76.6 \%)$ & $5(62.5 \%)$ & $28(73.7 \%)$ \\
Post-natal patient questionnaire completed $(n, \%)$ & $23(76.6 \%)$ & 1 & $28(73.7 \%)$ \\
Declined post-natal patient questionnaire $(n)$ & 0 & 7 & 1 \\
Agreed for post-natal interview $(n)$ & 14 & 7 & 21 \\
Underwent post-natal interview $(n)$ & 14 & 21 \\
\hline
\end{tabular}

SGH St. George's University Hospital, London, Medway Medway University Hospital, Kent

Table 3 Demographics of randomised participants

\begin{tabular}{|c|c|c|}
\hline Participant characteristics & Propess $(\boldsymbol{n}=20)$ & $\mathrm{CRB}(\boldsymbol{n}=18)$ \\
\hline \multicolumn{3}{|l|}{ Site } \\
\hline $\begin{array}{l}\text { SGH } \\
\text { Medway }\end{array}$ & $\begin{array}{l}16 \\
4\end{array}$ & $\begin{array}{l}14 \\
4\end{array}$ \\
\hline Mean age (SD) in years & $34.1(4.48)$ & $33.2(4.32)$ \\
\hline Mean height (SD) in $\mathrm{cm}$ & $166.62(6.25)$ & $171.59(7.1)$ \\
\hline Mean weight (SD) in kg & $71.84(11.9)$ & $65.96(11.0)$ \\
\hline Mean BMI (SD) in $\mathrm{kg} / \mathrm{m}^{2}$ & $25.72(2.9)$ & $22.37(3.3)$ \\
\hline \multicolumn{3}{|l|}{ Ethnicity } \\
\hline White & $15(75 \%)$ & $14(78 \%)$ \\
\hline Black & $1(5 \%)$ & $2(11 \%)$ \\
\hline Asian & $1(5 \%)$ & 0 \\
\hline Mixed & $1(5 \%)$ & $2(11 \%)$ \\
\hline Other & $1(5 \%)$ & 0 \\
\hline Not known & $1(5 \%)$ & 0 \\
\hline Mean gestational week (SD) at delivery & $41.71(0.61)$ & $41.63(0.53)$ \\
\hline \multicolumn{3}{|l|}{ Marital status } \\
\hline Married & $15(75 \%)$ & 7 (39\%) \\
\hline Cohabiting & $4(20 \%)$ & $8(44 \%)$ \\
\hline Single & $1(5 \%)$ & 0 \\
\hline Not recorded & 0 & $3(17 \%)$ \\
\hline \multicolumn{3}{|l|}{ Employment } \\
\hline Employed & $16(80 \%)$ & $18(100 \%)$ \\
\hline Unemployed & $2(10 \%)$ & 0 \\
\hline Not recorded & $2(10 \%)$ & 0 \\
\hline Nulliparous & $13(65 \%)$ & $12(67 \%)$ \\
\hline Parous & $7(35 \%)$ & $6(33 \%)$ \\
\hline
\end{tabular}

Data reported as mean (SD) or $n(\%)$ reference range at baseline, post-treatment and at follow-up. No uterine activity was detected at baseline in either of the two groups. The median Bishop score at study entry for both groups was unfavourable (Propess, 4; CRB, 3, Table 4). The clinical outcomes are shown in Table 5. The device (vaginal pessary or balloon catheter) was expelled in four (10.5\%) of women. Seven of the 38 participants could not go home after intervention (Table 5). Epidural use for labour analgesia was reported by $20 /$ $38(52.6 \%)$ of women. Nearly two thirds $(61 \%)$ of the women in the cervical balloon group had a spontaneous vaginal delivery, compared to $45 \%$ in the dinoprostone group. Delivery was by caesarean section in $14(36.8 \%)$ women. The rates of caesarean section were $33 \%(6 / 18)$ in the CRB group and 40\% (8/20) the Propess group.

Median gestational age at delivery was $41^{+6}$ weeks. Mean birthweights were similar between groups (Propess, 3688.7 gm; CRB, 3684.2 gm). All babies except one (37, 97.4\%) remained with the mother after delivery. No maternal or foetal deaths were recorded in this small sample.

The health economic outcomes are as follows: the AD-SUS service use questionnaire was easy to complete from patient records and the completeness of the data was excellent; $95 \%$ of participants had full service use data available at follow-up. Close monitoring over the data collection period ensured that we are confident that all relevant resources were included in the analysis. Full data at follow-up were available for 36 of the 38 randomised women. The costs of induction and readmission prior to delivery were estimated to be similar between the two randomised groups. Total costs were $£ 2880.82$ in the CRB group and $£ 3389.63$ in the Propess group (please see Table 5). This difference was accounted for by differences in mode of birth and it cannot be assumed from this small sample that a difference in mode of birth would be found with bigger numbers. 
Table 4 Maternal and foetal clinical parameters at baseline

\begin{tabular}{lll}
\hline Parameter & Propess $(\boldsymbol{n}=20)$ & CRB $(\boldsymbol{n}=18)$ \\
\hline Mean pulse (SD) in BPM & $83.4(10.35)$ & $80.8(9.63)$ \\
Mean systolic BP (SD) in mmHg & $118.7(9.71)$ & $117.2(12.40)$ \\
Mean diastolic BP (SD) in mmHg & $76.9(5.52)$ & $77.3(8.08)$ \\
Mean temperature (SD) in Celsius & $36.7(0.20)$ & $36.6(0.24)$ \\
Mean respiratory rate in breaths/min (SD) & $16.4(1.26)$ & $16.1(1.19)$ \\
Mean foetal heart rate (SD) in BPM & $137.6(12.18)$ & $141.3(12.83)$ \\
Median number (IQR) of contractions/10 min & $0(0$ to 0) & $0(0$ to 0) \\
Median Bishop score (IQR) & $4(3.0$ to 4.0) & $3.0(2.8$ to 4.3$)$ \\
\hline
\end{tabular}

\section{Women's willingness to be randomised}

Data were collected on reasons for declining participation. Overall, 93 women supplied decliners' data with 23 women completing a decliner's questionnaire and 70 women providing verbal responses. The most common reasons for declining to take part were as follows: preferring to have inpatient IOL $(n=22 ; 24 \%)$, declining IOL by any method before 42 weeks $(n=22 ; 24 \%)$ and preferring to have $\mathrm{PGE}_{2}$ pessary $(n=19 ; 20 \%)$.

\section{Discussion}

The number of women eligible for outpatient induction was much lower than anticipated. Reasons for underrecruitment included understaffing and not having the midwife(s) available to screen women every day and women's preference to a method, in particular prostaglandin pessary. Suitability of outpatient induction is dependent on the local criteria. Units with restrictive criteria will have a limited number of women deemed suitable for outpatient induction of labour. A widening of inclusion criteria may increase numbers of potential participants. The study is not feasible using existing criteria and further modifications to the eligibility criteria for outpatient IOL would be needed to make an RCT feasible. At Medway Hospital, consenting women underwent an artificial rupture of membranes (ARM) if feasible rather than entry into the trial due to a policy change. This led to a reduction in the pool of possible participants limiting recruitment. Therefore, further recruitment at Medway Hospital was stopped. Choice of another suitable unit where consenting women were allowed to participate in the study could have helped with recruitment.

Approximately a third of all eligible women in this study $(84 / 230,36.5 \%)$ were prepared to participate in a trial where the method of induction of labour in the outpatient setting (dinoprostone or CRB) is chosen at random. Participant numbers were limited further by clinical factors, particularly women going into labour spontaneously before the CR method was inserted, and a smaller number of women withdrawing after recruitment. A previous study by Henry et al. [4] exploring outpatient Foley catheter versus inpatient prostaglandin E2 gel for induction of labour reported that out of the 262 women found eligible for inclusion, 101 (38.5\%) agreed and were randomised. This rate is very similar to that observed in this study, although the Henry study took place in Australia.

Outpatient induction of labour is not common in the UK. In a survey of outpatient IOL [13], a postal questionnaire was sent to 210 NHS consultant-led obstetric units within the UK, of which $78 \%$ responded. Only $17.6 \%$ of units reported that they currently or soon will provide outpatient IOL. The rate of use may have increased since this survey but routine data are not available. Outpatient IOL may benefit the working of midwives as well. A survey exploring the impact of outpatient IOL on midwives' work found that their job satisfaction either increased or was unchanged in an overwhelming majority (93\%) of respondents [14].

More than one half of those women approached in this study declined to participate. The most common reasons for declining to take part were preferring to have inpatient IOL, declining IOL by any method before 42 weeks, plus preferring to have $\mathrm{PGE}_{2}$ pessary. Since a trial of this nature cannot be blinded, there is also a possibility that agreement to continue in a trial may be skewed by women's prior attitudes. This is reflected in the reasons given for declining, and CRB was not standard practice in these services; preference for this method could equally have been a motivator for participation. Mechanical methods have been reported as being safer than prosaglandins for labour induction [15]. However, that study was published relatively recently and the findings not widely disseminated. Improved information provision may remove this obstacle and provide eligible women with a wider choice.

A majority of women who were randomised received the intended intervention and were able to go home. Delivery was by caesarean section in $14(36.8 \%)$ women. All babies were born with good condition and only one baby did not stay with the mother after delivery. Severe complications were reported only in a small minority (Table 5). One woman from the CRB group experienced severe post-partum haemorrhage $(2.1 \mathrm{~L})$ and one woman from the Propess group underwent a category 1 caesarean section for antepartum haemorrhage. 
Table 5 Clinical and health economic outcomes

\begin{tabular}{|c|c|c|}
\hline Participant outcome & Propess $(\boldsymbol{n}=20)$ & CRB $(\boldsymbol{n}=18)$ \\
\hline \multicolumn{3}{|l|}{ Intervention expelled } \\
\hline No & $15(75 \%)$ & $14(78 \%)$ \\
\hline Yes & $3(15 \%)$ & $1(6 \%)$ \\
\hline Missing & $2(10 \%)$ & $3(16 \%)$ \\
\hline \multicolumn{3}{|l|}{ Return for admission } \\
\hline Agreed time next morning & $5(25 \%)$ & $4(22 \%)$ \\
\hline Labour & $10(50 \%)$ & $5(28 \%)$ \\
\hline Participant never went home following intervention & $3(15 \%)$ & $4(22 \%)$ \\
\hline Missing & $2(10 \%)$ & $5(28 \%)$ \\
\hline \multicolumn{3}{|l|}{ Epidural use } \\
\hline No & $8(40 \%)$ & $9(50 \%)$ \\
\hline Yes & $12(60 \%)$ & $8(44 \%)$ \\
\hline Missing & 0 & $1(6 \%)$ \\
\hline Live birth & $20(100 \%)$ & $18(100 \%)$ \\
\hline \multicolumn{3}{|l|}{ Mode of birth } \\
\hline SVD & $9(45 \%)$ & $11(61 \%)$ \\
\hline Caesarean section in labour & $6(30 \%)$ & $2(11 \%)$ \\
\hline No labour caesarean section & $2(10 \%)$ & $4(22 \%)$ \\
\hline Instrumental delivery & $3(15 \%)$ & $1(6 \%)$ \\
\hline Median estimated blood loss in ml (IQR) & $320.0(200.0$ to 675.0$)$ & 600.0 (225.0 to 1145.0$)$ \\
\hline Mean birthweight in gm (SD) & $3688.7(310.00)$ & $3684.2(293.33)$ \\
\hline Boy & $14(70 \%)$ & $6(33 \%)$ \\
\hline Girl & $6(30 \%)$ & $11(61 \%)$ \\
\hline Not recorded & 0 & $1(6 \%)$ \\
\hline 5 min Apgar score median (IQR) & $10.0(10.0$ to 10.0$)$ & $10.0(10.0$ to 10.0$)$ \\
\hline Head circumference in cm (mean, SD) & $35.2(1.54)$ & $35.1(1.36)$ \\
\hline \multicolumn{3}{|l|}{ Where did the baby go? } \\
\hline To mother & $20(100 \%)$ & $16(89 \%)$ \\
\hline NICU admission & 0 & $1(6 \%)$ \\
\hline Missing & 0 & $1(6 \%)$ \\
\hline \multicolumn{3}{|l|}{ Any adverse event experienced } \\
\hline Mother alone & $8(40 \%)$ & $6(33 \%)$ \\
\hline Baby alone & $1(5 \%)$ & $3(17 \%)$ \\
\hline Both mother and baby & $4(20 \%)$ & $2(11 \%)$ \\
\hline None & $7(35 \%)$ & $7(39 \%)$ \\
\hline \multicolumn{3}{|l|}{ Severe adverse event } \\
\hline None & $18(90 \%)$ & $17(94 \%)$ \\
\hline Mother alone & $1+(10 \%)$ & $1(6 \%)$ \\
\hline Baby alone & 0 & 0 \\
\hline Both mother and baby & 0 & 0 \\
\hline Maternal death & 0 & 0 \\
\hline Foetal/neonatal death & 0 & 0 \\
\hline Health economic outcomes & Propess $(n=19)$ & CRB $(n=17)$ \\
\hline Cost of induction and readmission prior to delivery, mean (SD) & $135.47(206.56)$ & $127.29(198.33)$ \\
\hline
\end{tabular}


Table 5 Clinical and health economic outcomes (Continued)

\begin{tabular}{lll}
\hline Participant outcome & Propess $(\boldsymbol{n}=20)$ & CRB $(\boldsymbol{n}=18)$ \\
\hline Cost of delivery, mean (SD) & $3254.16(965.10)$ & $2753.53(712.31)$ \\
Total cost, mean (SD) & $3389.63(1023.94)$ & $2880.82(717.04)$ \\
\hline
\end{tabular}

tOne mother experienced two severe adverse events (haemorrhage and infection)

Not all women who desired to go home could go home. Twenty-nine percent of the participating women could remain home overnight in this study. Wilkinson et al. [7] reported that less than half the women participating in an RCT comparing inpatient versus outpatient cervical ripening could remain at home overnight. However, all women had received vaginal prostaglandin $\mathrm{E}_{2}$ gel [7].

\section{Conclusions}

The study is not feasible using existing eligibility criteria for outpatient induction of labour (IOL). Further modifications to the eligibility criteria for outpatient IOL improved provision of information on safety and better availability of research staff may be helpful to make such a trial feasible. The trial procedures were acceptable to women who participated. Although the sample size of this feasibility trial was limited, no major adverse effects attributible either to the setting or the method for induction of labour were recorded. Service use data can be collected in a vast majority of participants.

\section{Acknowledgements}

The authors acknowledge the contribution of the members of the Trial Steering Committee (TSC): Professor Lucy Chappell (chair), Professor Andrew Weeks and Dr. Louise Marston. We gratefully acknowledge Dr. Sharon Griffin, the principal investigator at Medway Hospital, for her efforts with patient recruitment.

\section{Authors' contributions}

AB: conceived the idea, secured funding, conducted the study, interpreted the results, wrote the manuscript. CM: secured funding, supervised qualitative research, interpreted the results. BB: secured funding, led health economics research, interpreted the results. GC: conducted the study. RC: conducted qualitative research, interpreted the results. RG: secured funding, patient representative, interpreted the results. SL: secured funding, interpreted the results. PS: secured funding, trial statistician, interpreted the results. All the authors critically appraised and approved the manuscript.

\section{Funding}

Funding was received from National Institute for Health Research (NIHR), research for patient benefit scheme (PB-PG-0815-20022). The views expressed in this publication are those of the authors and not necessarily those of the NHS, NIHR, the Department of Health or Social Care.

\section{Availability of data and materials}

The trial essential documents along with the trial database will be archived in accordance with the Sponsor (Joint Research and Enterprise Office, St. George's, University of London) SOP JREOSOP0016. The agreed archiving period for this trial will be 15 years. The data will be available for sharing by contacting the chief investigator. Publication policy has been documented in the project protocol

\section{Ethics approval and consent to participate}

The trial was approved by the East of England - Cambridgeshire and Hertfordshire Research Ethics Committee (17/EE/0295)

\section{Consent for publication}

N/A

\section{Competing interests}

None

\section{Author details}

${ }^{1}$ Fetal Medicine Unit, St. George's University Hospital Foundation Trust, Blackshaw Road, London SW17 OQT, UK. 'St George's, University of London, London, UK. ${ }^{3} \mathrm{UiT}$ : The Arctic University of Norway, Tromsø, Norway. ${ }^{4}$ King's

Clinical Trials Unit, London, UK. ${ }^{5}$ City, University of London, London, UK.

${ }^{6}$ Maternity Voices Partnership Chair, St. George's University Hospital

Foundation Trust, London, UK

Received: 7 February 2020 Accepted: 3 August 2020

Published online: 15 August 2020

\section{References}

1. NHS Digital SCT. NHS maternity Statistics. 2017-18. https:/files.digital.nhs.uk/C3/4 7466E/hosp-epis-stat-mat-summary-report\%202017-18.pdf. Published 2018. Accessed 23/09/2019.

2. Kelly AJ, Alfirevic Z, Ghosh A. Outpatient versus inpatient induction of labour for improving birth outcomes. Cochrane Database Syst Rev. 2013;11:CD007372.

3. de Vaan MD, Ten Eikelder ML, Jozwiak M, et al. Mechanical methods for induction of labour. Cochrane Database Syst Rev. 2019;10:CD001233.

4. Henry A, Madan A, Reid R, et al. Outpatient Foley catheter versus inpatient prostaglandin E2 gel for induction of labour: a randomised trial. BMC Pregnancy Childbirth. 2013;13:25

5. Jozwiak M, Oude Rengerink K, Benthem M, et al. Foley catheter versus vaginal prostaglandin E2 gel for induction of labour at term (PROBAAT trial): an open-label, randomised controlled trial. Lancet. 2011;378(9809):2095-103.

6. Pennell CE, Henderson JJ, O'Neill MJ, McChlery S, Doherty DA, Dickinson JE. Induction of labour in nulliparous women with an unfavourable cervix: a randomised controlled trial comparing double and single balloon catheters and PGE2 gel. BJOG. 2009;116(11):1443-52.

7. Wilkinson C, Bryce R, Adelson P, Turnbull D. A randomised controlled trial of outpatient compared with inpatient cervical ripening with prostaglandin E(2) (OPRA study). BJOG. 2015;122(1):94-104.

8. Balloon Catheter Versus Propess for Labour Induction (PROBIT-F). https:// clinicaltrials.gov/ct2/show/NCT03199820. Accessed 23/09/2019.

9. No authoirs listed. NIHR rfpb program guidance. https://www.nihr.ac.uk/ documents/nihr-research-for-patient-benefit-rfpb-programme-guidance-onapplying-for-feasibility-studies/20474). Accessed 24/05/2020.

10. Teare MD, Dimairo M, Shephard N, Hayman A, Whitehead A, Walters SJ. Sample size requirements to estimate key design parameters from external pilot randomised controlled trials: a simulation study. Trials. 2014;15:264.

11. Wilkinson EL, O'Mahen HA, Fearon P, et al. Adapting and testing a brief intervention to reduce maternal anxiety during pregnancy (ACORN): study protocol for a randomised controlled trial. Trials. 2016;17:156.

12. Eldridge SM, Chan CL, Campbell MJ, et al. CONSORT 2010 statement: extension to randomised pilot and feasibility trials. BMJ. 2016;355:15239.

13. Sharp AN, Stock SJ, Alfirevic Z. Outpatient induction of labour in the UK: a survey of practice. Eur J Obstet Gynecol Reprod Biol. 2016;204:21-3.

14. Turnbull D, Adelson P, Oster $C$, et al. The impact of outpatient priming for induction of labour on midwives' work demand, work autonomy and satisfaction. Women Birth. 2013;26(3):207-12.

15. Du YM, Zhu LY, Cui LN, Jin BH, Ou JL. Double-balloon catheter versus prostaglandin E2 for cervical ripening and labour induction: a systematic review and meta-analysis of randomised controlled trials. BJOG. 2017;124(6): $891-9$.

\section{Publisher's Note}

Springer Nature remains neutral with regard to jurisdictional claims in published maps and institutional affiliations. 